\title{
Similarities Found in Neurological Disorders Based on Mutated Genes
}

\author{
Amanda Chen and Melanie Mendoza \\ Newburgh Free Academy, amanda.netsci@gmail.com
}

\begin{abstract}
According to the UN World Health Organization, nearly one in every six of the world's population is affected by a neurological disorder. This research study focused on neural networks and analyzed the connections between neurological disorders defined by similar single nucleotide polymorphisms (SNP). By utilizing the Genome-wide Association studies, SNPs associated with a select group of neurological disorders were identified. Drug molecules that target these SNPs were gathered from the DrugBank. The results showed that Schizophrenia and Bipolar disorder were the most genetically similar based on the SNPs associated with them. Also, Schizophrenia had the highest number of SNPs associated with it as well as the highest betweenness rank. The gene TRNAI25 was the gene found among four of the disorders and it is implicated in various biological roles such as coding, decoding, regulation, and expression of genes. The drug, Felodipine, targets two SNPs, one associated with Schizophrenia and the other with Bipolar Disorder. Neurological disorders are being visualized through patient CT or MRI scans; however, this study focused on the shared SNPs in different neurological disorders generated from preexisting data. With this project, genetic similarities were found within a select group of neurological disorders and may further biological research in neurological disorders through the use of network science.
\end{abstract}

Index Terms: ADHD, Alzheimer's Disease, Autism, Bipolar Disorder, Epilepsy, high school research, K-12, mutated genes, neurological disorders, network science, neural network analysis, Parkinson's Disorder, Schizophrenia

\section{Introduction}

Throughout the 2014-15 academic year two students, Melanie Mendoza and Amanda Chen from Newburgh Free Academy in Newburgh, NY worked with teacher, Veronica Dunham, on an independent research project as part of NetSci High. NetSci High is a program that brings students and teachers together with researchers to learn the basic tools of network analysis at a NetSci High summer workshop. Students then choose an application to work on throughout the academic year mentored by researchers. This team collaborated with faculty from the Network Science Center at West Point. The project focused on analyzing neural disorder networks and the connection between neurological disorders through similar mutated genes. They also studied connections between similar drug molecules used to target the specific mutated genes connected to this small group of neurological disorders. Using this multimodal network they developed three research questions:

1. Which neurological disorders are most similar based on the shared mutated genes between them?

2. Is there a specific mutated gene that is shown to be associated with several disorders?

3. Is there a certain drug molecule that is targeted towards several mutated genes?

Our goal is to research the linkage between different neurological disorders using this new perspective and to find specific drug molecules that can treat multiple neurological disorders. 


\section{Background}

This team choose to look at disorders characterized as non-communicable, genetically predispositioned malfunctions in the central nervous system of the brain. The students did extensive background research on seven disorders; ADHD, Alzheimer's disease, Autism, Bipolar Disorder, Epilepsy, Parkinson's Disorder, and Schizophrenia, to formulate a hypotheses of similarities, common mutated genes, and links between drug molecules. According to the research, Schizophrenia as well as Bipolar Disorder show the most similarities in symptoms; therefore, we predicted that those two disorders would be the most genetically similar. People with Schizophrenia have been shown to have abnormal levels of naturally occurring neurotransmitters and hormones such as dopamine and glutamate causing symptoms such as delusions or false voices in their head. Also, scientists have seen abnormalities in brain structure and the central nervous system in people with Schizophrenia compared with healthy people. People with Bipolar disorder tend to have unusual shifts in mood, energy, activity levels. Scientists have seen abnormalities in brain structure and the central nervous system in people with Bipolar disorder compared with healthy people. We also predicted that because Alzheimer's disease and Parkinson's disorder both involve the slow deterioration of neurons over time, they would also be genetically similar. Alzheimer's is characterized by the loss of neurons in conjunction with the presence of oxidative stress, axonal dystrophy, mature senile plaques and neurofibrillary tangles while Parkinson's is caused by the absence or very low amounts of dopamine due to the slow deterioration of neurons. The other three disorders include: Epilepsy, Autism, and ADHD. Epilepsy, due to an imbalance in neurotransmitters and nerve signaling, is characterized by unpredictable seizures and is the fourth most common neurological disorder. ADHD, most common in young children, is caused by abnormally thin brain tissue that usually thickens over time, therefore, allowing the symptoms to improve. Autism is a complex neurological disorder that results in learning disabilities and social impairments. It is thought to be developed during fetal growth when there is not enough oxygen reaching the brain causing it to become deformed. Researching the biological aspects behind these seven disorders was imperative to understanding the results of our research.

\section{Process}

Students obtained their data of disorder-gene interactions from the Genome-wide Association Studies (GWAS), a catalog compiling the different SNPs (mutated nucleotide base in DNA) that correlate to different disorders scientists have researched over time. When SNPs occur within a gene or in a regulatory region near a gene, they may play a more direct role in disease by affecting the gene's function. DrugBank was used to find the drug molecules that can be targeted towards a specific gene. Each gene found in the GWAS was searched in DrugBank as a target which then was used to find the drug molecules linked to that specific gene. The data was then imported into Gephi to create the visualization and calculate centrality measures. From there, students analyzed the degree of each gene to determine if there is one specific gene that is common in many of the disorders. The same was done with the drug molecules to determine how versatile it can be towards several genes.

\section{Results}

One of our original research questions was if there was one specific mutated gene that was found among many of the neurological disorders. TRNAI25 was the only SNP with the highest degree of four. The TRNAI25 gene was found in patients with ADHD, Parkinson's disorder, Bipolar Disorder, and Schizophrenia. All four of these disorders were found to be caused by structural abnormalities in the brain as well as an imbalance of certain neurological transmitters and hormones. There is no concrete abnormality that is shared between these four disorders because the same abnormalities can be 
seen in patients with disorders that are not associated with TRNAI25 (Epilepsy, Autism, and Alzheimer's).

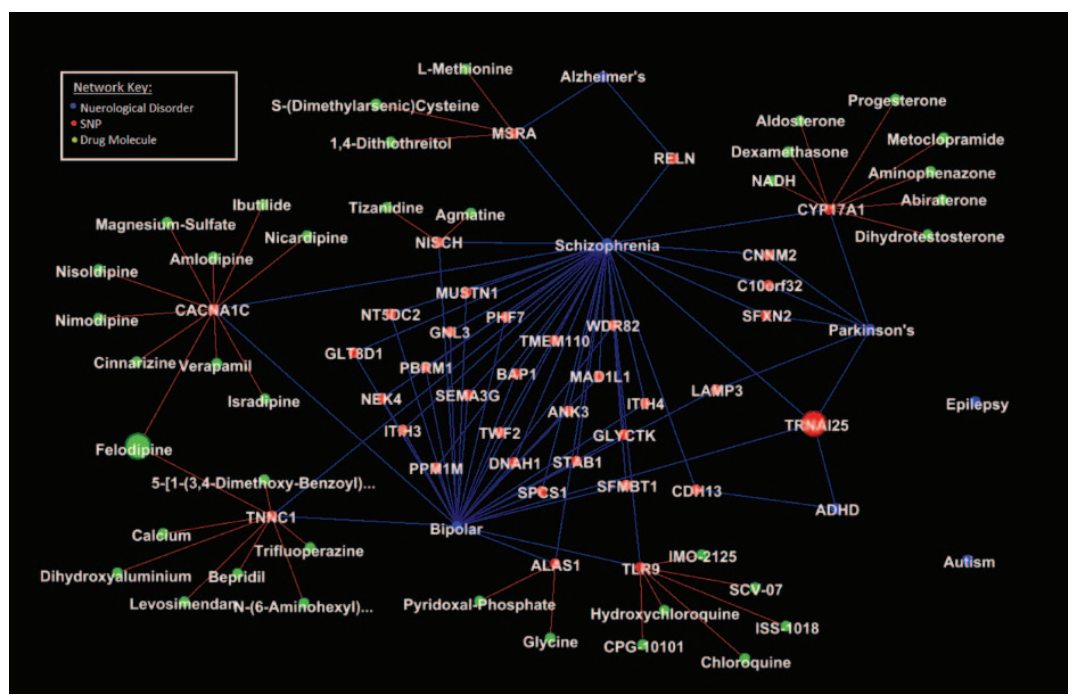

Epilepsy and Autism were found to be isolates, meaning they did not share any mutated genes with other disorders. It was found that Schizophrenia and Bipolar have the most commonly mutated genes of all the disorders, with the highest betweenness measure. The brain's prefrontal cortex in adults with Bipolar disorder tends to be smaller and less functional, than a mentally healthy adult. This structure and its connections to other parts of the brain mature during adolescence, suggesting that abnormal development of this brain circuit may account for why the disorder tends to emerge during teenage years. Schizophrenia is known to cause abnormal levels of naturally occurring neurotransmitters and also abnormalities in brain structure.

The drug molecule Felodipine was found to target two genes, CACNA1C and TNNC1 which are associated with Bipolar and Schizophrenia. Felodipine is used to treat high blood pressure. Felodipine prevents calcium from being released within the muscle cells of the small arteries and thereby causes the muscles to relax and the arteries to dilate or expand. High blood pressure within the brain can be fatal to a person because the brain has no room to expand within the skull. Felodipine may be used to treat symptoms of these disorders, in this case high blood pressure, but does not target the SNP itself to stop or slow down the development of the disorder.

\section{Future Work}

Why is it that Epilepsy and Autism have not yet been found to share common SNP's with other neurological disorders? Why was Schizophrenia found to have the most mutated genes? Is it because there many types of Schizophrenia? In order to carry this project to the next level, we would need to research more about the history of these disorders and look for correlations to the amount mutated genes one has. 